\title{
Timescales of self-healing in human bone tissue and polymeric ionic liquids
}

1 Johanna Akbarzadeh

Faculty of Physics, University of Vienna, Vienna, Austria

2 Stephan Puchegger

Faculty of Physics, University of Vienna, Vienna, Austria

3 Anja Stojanovic

Faculty of Natural Sciences II, Chair of Macromolecular Chemistry, Martin-Luther University Halle-Wittenberg, Halle, Germany

4. Helmut O. K. Kirchner

Institute of New Materials GmbH, Saarbrücken, Germany
5 Wolfgang $\mathrm{H}$. Binder

Faculty of Natural Sciences II, Chair of Macromolecular Chemistry, Martin-Luther University Halle-Wittenberg, Halle, Germany

$6 \quad$ Sigrid Bernstorff

Elettra-Sincrotrone Trieste, S.C.p.A., Area Science Park, Basovizza, Trieste, Italy

7 Peter Zioupos

Biomechanics Laboratories, Cranfield University, Shrivenham, UK

8 Herwig Peterlik*

Faculty of Physics, University of Vienna, Vienna, Austria
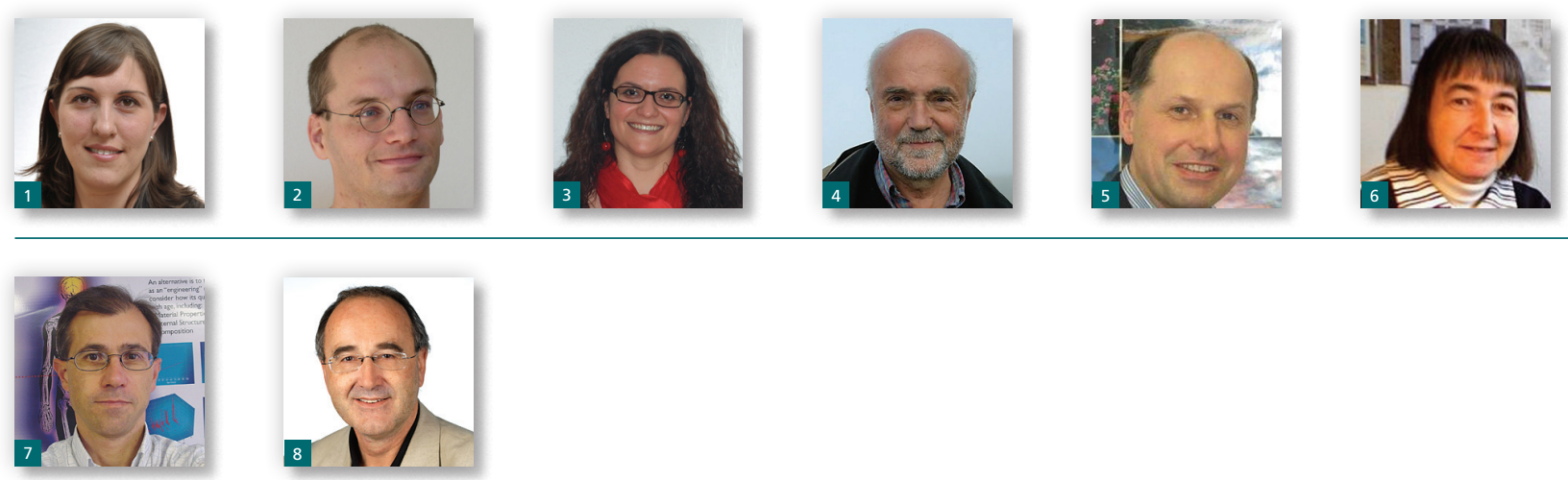

Strain (stress-free) relaxation in mechanically prestrained bone has a time constant of $75 \mathrm{~s}$. It occurs by a reorganization of the proteoglycan-glycoprotein matrix between collagen fibers, which requires ionic interactions. Dissolving and relinking the ionic bonds is thus an important tool of nature to enable plastic deformation and to develop self-healing tissues. A way to transfer this approach to technical materials is the attachment of ionic end groups to polymeric chains. In these classes of materials, the so-called polymeric ionic liquids, structural recovery of thermally disorganized material is observed. A time constant between minutes and a week could be achieved, also by ionic rearrangement. The same mechanism, rearrangement of ionic bonds, can lead to vastly different relaxation times when the ionic interaction is varied by exchange of the cationic end groups or the anions.

\section{Introduction}

A striking feature of biological tissues is invariably the presence of a hierarchical structure, ${ }^{1,2}$ and the ability of self-healing, even of critically damaged material by cellular activity and the formation of intermediate tissue. ${ }^{2}$ This formation is induced by chemical signals, involving inflammation, which leads to wound closure and matrix remodeling. ${ }^{3}$ Self-healing takes place not only at the tissue level but also at the molecular level, probably by breaking and repairing of 'sacrificial bonds' under load. ${ }^{4-6}$ In the hierarchical structure, ${ }^{6-8}$ the self-healing properties are connected to specific structural levels.

For the molecular level, in situ experiments using synchrotron radiation by Gupta et al..$^{9}$ have convincingly shown that plastic deformation of bone occurs in the proteoglycan-glycoprotein matrix (about 5 vol\%) between collagen fibrils, hitherto referred to as pggp-matrix. In macroscopic mechanical tests, ${ }^{10}$ bovine fibrous-lamellar bone was strained at various temperatures and 
various strain rates. An interpretation in terms of a thermally activated process led to an activation enthalpy of $H=1.1 \mathrm{eV}$ and an activation volume of $V=0.6 \mathrm{~nm}^{3} .{ }^{10}$ The high activation enthalpy and small activation volume rule out the participation of hydrogen bonds, which were considered earlier as structural origin of bone plasticity. ${ }^{11}$ These magnitudes suggest that bone plasticity occurs by the breaking of calcium-mediated (ionic) bonds between polyelectrolytic proteins (such as osteopontin, fetuin A) and proteoglycans (such as decorin) in the interfibrillar matrix. ${ }^{4}$ The $\mathrm{Ca}^{2+}$-mediated liaison is broken according to the below reaction.

\section{Protein $/ \mathrm{Ca}^{2+} /$ protein $\rightarrow$ protein $/ \ldots . / \mathrm{Ca}^{2+} / \ldots . /$ protein}

This ionic interaction corresponds to breaking and reforming of 'sacrificial bonds' observed by Fantner et al. ${ }^{4,6}$ It can also be called the formation of two structural defects, the two unsaturated bonds /.../, on the right-hand side of the Equation 1. The transition from the energetically favourable left side of Equation (1) to the unfavourable right side is forced by the applied stress. After unloading, the possibility of the reverse reaction (1) leads to a self-healing of the interfibrillar matrix by a re-establishment of these forcefully broken bonds. Essential for this self-healing at the molecular level is an ionic interaction (bonds that can be broken and linked again). ${ }^{12}$ The distance between the two partners has to be sufficiently small (in the nanometer range) to ensure a high probability of linking two chains together ionically.

Observation of self-healing in biological tissues inspired the development and synthesis of self-healing synthetic polymers, in which damage triggers the repair process. ${ }^{13,14}$ Self-healing in polymers can occur by molecular interdiffusion, recombination of chain ends, reversible bond formation, living polymers with active chain ends capable of resuming polymerization, selfhealing by nanoparticles, self-healing microcapsules, to name but a few. ${ }^{14}$ As dissolving and restoring the links by ionic interaction is a key issue in biological materials such as bone tissue, the introduction and control of additional ionic interaction in polymeric chains could foster the development of self-healing technical materials. In the following, we present the observation of self-healing for a new material class, polymeric ionic liquids (POILS). ${ }^{15-17}$ They combine polymeric chains with ionic end groups, where the enhanced ionic interaction can lead to selfrepair. Whereas ILs are usually salts with a melting point below $100^{\circ} \mathrm{C}$, POILs are usually solids. Besides polymeric chains, POILS contain bulky organic cations such as imidazolium, pyridinium, ammonium or phosphium cations ${ }^{18-21}$ together with organic and inorganic anions. Heat treatment of POILS destroys their long-range order, and we study the kinetics of subsequent self-healing. As an example, we investigate POILs with the polymeric compound polyisobutylene (PIB), being synthesized by a living carbocationic polymerization (LCCP) and a subsequent azid-alkyne 'click'-reaction for the attachment of the ionic groups. A detailed description of the molecular design, the synthesis process and properties of the POILs was published recently. ${ }^{22,23}$

\section{Materials and methods}

We followed the structural reorganization in a biological tissue (bone) and a technical material (POILs), by in situ small-angle (SAXS) and wide-angle X-ray scattering (WAXS), after they had been strained to fracture (bone) or destruction of long-range order by heating (POILs).

\subsection{Bone}

Bone specimens were cut from 6-cm-long mid-diaphyseal cylindrical segments from femurs of male donors, deceased at the age of 42 92 years from causes unrelated to their bones. The preparation was similar to Zioupos et al. ${ }^{24,25}$ : The specimens were kept at $-20^{\circ} \mathrm{C}$ in airtight plastic containers wrapped in cloth soaked in Ringers solution. The specimens were grinded at low speed to final dimension (approximately $20 \times 2 \times 0.5 \mathrm{~mm}^{3}$ ), kept cool until the experiment and then tested at room temperature (RT) in a tensile testing stage, equipped with a $1.25 \mathrm{kN}$ load cell. The specimens were kept wet during the test using a wick wetted with Ringer's solution. The structural change was followed in situ by X-rays from a synchrotron radiation source (SAXS-beamline, ELETTRA, Trieste). The X-ray energy was $16 \mathrm{keV}$, which corresponds to a wavelength $\lambda=0.79 \AA$. In the SAXS region of reciprocal space, the $d$-period of the collagen molecules gives rise to an axial diffraction pattern, from which the third diffraction peak $d_{003}$ was evaluated. In bone, this regular interval corresponds to the distance of mineral crystals, whose nucleation and crystal formation is triggered by collagen or by other noncollageneous proteins. ${ }^{2}$ The position of the diffraction peak and subsequently the strain were determined by radial integration of the 2D (two dimension) patterns in $70^{\circ}$-wide pie-shaped sectors and fitted with Gaussian functions. For the position and the strain of the mineral particles, the shift of the (112)-reflection with strain was used and recorded with a one-dimension detector in the WAXS region.

Our experiments resemble those of Gupta et al. on bovine femural bone, ${ }^{9}$ but we focus on the relaxation aspect. In order to avoid any problems with machine stiffness and mounting, notoriously disturbing in relaxation experiments, we chose to look at the stressfree state after fracture. The typical strain to fracture was about 5\%, as it is usual in bone (most of which is known to be taken up by the pggp-matrix). ${ }^{9}$

\subsection{POILS}

As POILS, we chose PIB-based ILs (PIB-ILs) for biocompatibility, high hydrophobicity and chain mobility. Three different ILs (methylimidazolium, pyrrolidinium and triethylammounium) served 
Bioinspired, Biomimetic and Nanobiomaterials

Volume 3 Issue BBN3
Timescales of self-healing in human bone tissue and polymeric ionic liquids Akbarzadeh et al. as cations. The POILs were synthesized by a LCCP and a subsequent 'click' reaction to attach the ionic end groups to the polymeric chains. The synthetic process of the specimens PIB-IL $3 a-3 c$ is described in Ref. ${ }^{22}$. All three use bromide as anion but differ in the cation. With these materials, we intend to show the importance of the ionic end group on the timescales of structural reorganization. The influence of the anion can be investigated after an anion exchange: In our case, bromide was exchanged with bis(trifluoromethylsulfonyl)imide lithium salt (anion $\mathrm{Tf}_{2} \mathrm{~N}$ ). ${ }^{23}$ This synthesis step leads, together with the respective ionic end group, to the materials PIB-IL 6a-6c. Figure 1 shows a scheme of the structure of these POILs, differing in their ionic interaction as well by the cation and the anion.

The structural reorganization of POILs was followed by in situ SAXS in a laboratory equipment (Bruker Nanostar). SAXS patterns were collected with a 2D position sensitive detector (VÅNTEC 2000) for typically $15 \mathrm{~min}$. Before each measurement, the samples were heated stepwise (heating rate $5^{\circ} \mathrm{C} / \mathrm{min}$ ) in a specially designed $\mathrm{X}$-ray transparent furnace to a desired temperature and kept at this temperature for $5 \mathrm{~min}$ to ensure thermal equilibrium. Then, the samples were cooled to RT (cooling rate approximately around half the heating rate), and the structural recovery was followed by measurements in regular time intervals (in the range from parts of an hour to a week). The distance between sample and detector was $108 \mathrm{~cm}$, leading to an accessible $q$-range from $0 \cdot 1$ to $2 \cdot 8 \mathrm{~nm}^{-1}$. From the radially averaged SAXS patterns, the (100) peak of the (hexagonal or cubic) structure was identified and fitted with a Lorentzian function. The lattice constant was calculated from the peak maximum.

\section{Results}

\subsection{Bone}

Immediately after fracture, the strain of the collagen fibrils relaxes exponentially,

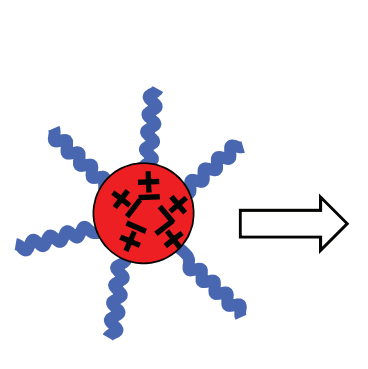

(a)

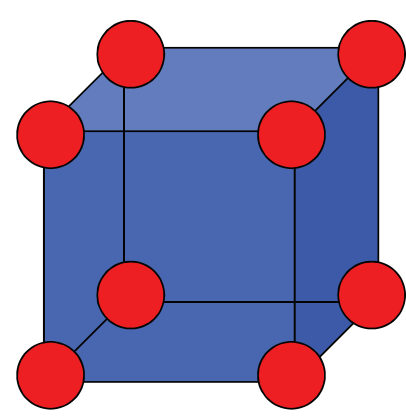

(b)
Figure 1. Structure and synthesis of non-symmetric ionic liquidfunctionalized PIBS 22,23: (a) basic unit; (b) simple cubic arrangement of basic units; (c) variation in cation leads to indices a-c, bromide as 2a.

$$
\varepsilon=\varepsilon_{0} \exp [-r t]
$$

corresponding to a first-order reaction

$$
\text { 2b. } \frac{\mathrm{d} \varepsilon}{\mathrm{d} t}=-r \varepsilon
$$

In the Kelvin-Voigt model of a solid undergoing reversible, viscoelastic strain, the stress $\sigma$ is proportional to strain $\varepsilon$ and strain rate $\mathrm{d} \varepsilon / \mathrm{d} t$,

3. $\sigma=E \varepsilon+\eta \frac{\mathrm{d} \varepsilon}{\mathrm{d} t}$

The rate of recovery is $r=E / \eta$, because the external stress $\sigma=0 \mathrm{MPa}$ in our experiments. For creep of metals, Equation $2 \mathrm{~b}$ is known as the Bailey-Orowan equation. ${ }^{26}$ Figures 2 and 3 show the decay of the strain in the collagen fibrils and the mineral crystals after fracture. This is not the usual creep recovery experiment, where the strain is held constant and the stress decays by plasticity. To the contrary, we observe spontaneous strain changes under no applied stress.

Figure 2(a)-2(c) shows the loading program (increase in stress up to fracture), the change in distance of the third-order diffraction peak from the $d$-period of the collagen molecules and the (112)-reflection of the mineral crystals, respectively. The quantity $[\mathrm{d}\{t\}-\mathrm{d}\{t=0\}] /[\mathrm{d}\{t=0\}]=\varepsilon[t]$ is the time-dependent strain. The figure shows the data of a human male femur (age 46 years, deceased for reasons unrelated to any bone disease). Between $t=$ $-600 \mathrm{~s}$ and $t=0 \mathrm{~s}$, the specimen was strained at a rate of about $\mathrm{d} \varepsilon /$ $\mathrm{d} t=10^{-5} \mathrm{~s}^{-1}$, it ruptured at $t=0 \mathrm{~s}$ and relaxed afterward according to $d[t]=d[t=0] \exp [-r t]$, with $1 / r=50 \mathrm{~s}$ for the collagen and less

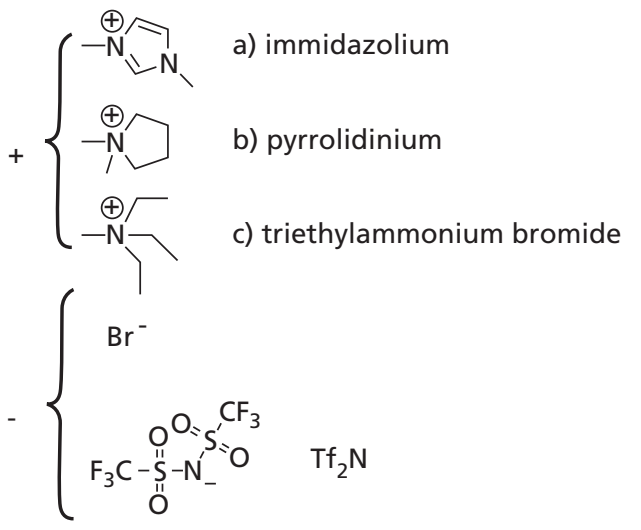

(c)

anion to sample series $3 a-3 c$ and $\mathrm{TF}_{2} \mathrm{~N}$ anion to series $6 \mathrm{a}-6 \mathrm{c}$. PIB, polyisobutylene 
Bioinspired, Biomimetic and Nanobiomaterials Volume 3 Issue BBN3
Timescales of self-healing in human bone tissue and polymeric ionic liquids Akbarzadeh et al.

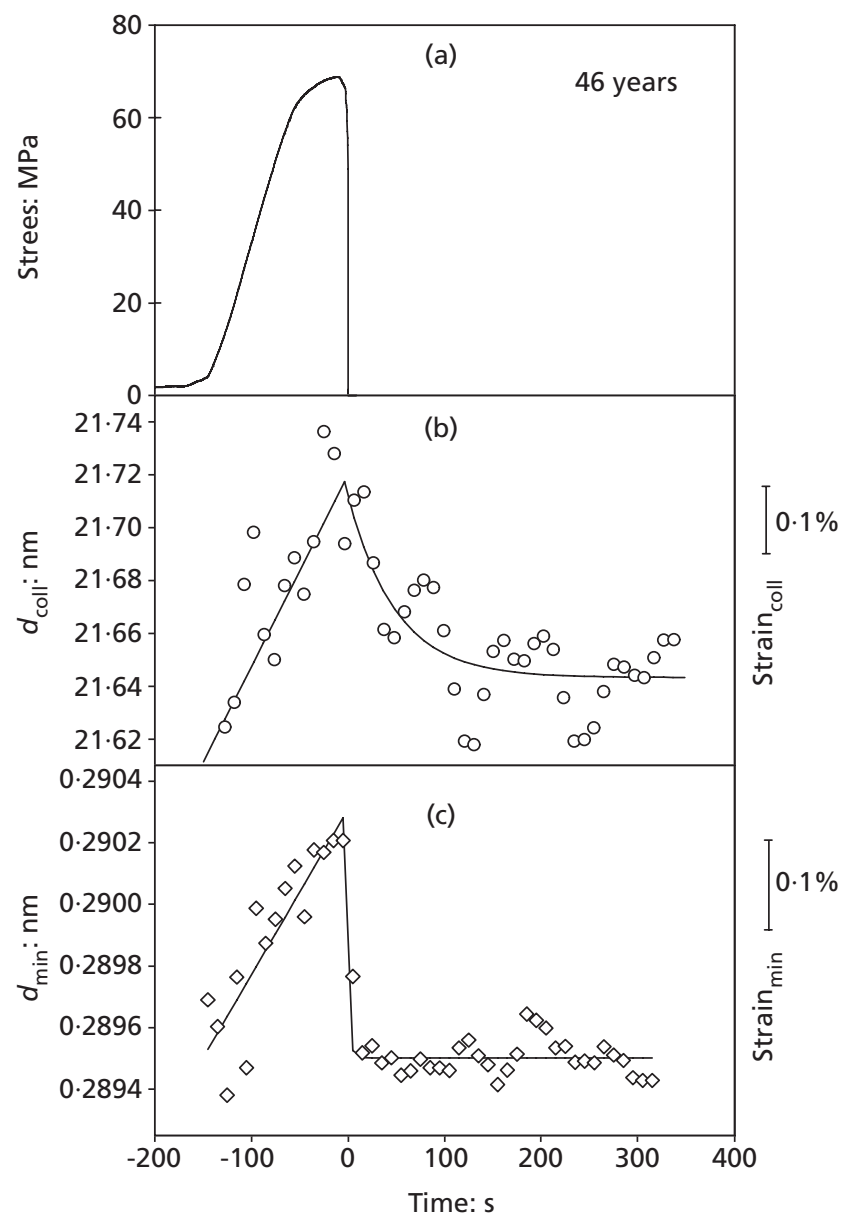

Figure 2. (a) Loading history (stress as function of time), (b) position of third reflection $d_{003}$ in real space from the $d$-period of collagen and (c) of (112)-reflection from the mineral in dependence on time and associated strains, respectively

than $10 \mathrm{~s}$ (the exposure time for an X-ray image in the synchrotron radiation source was $10 \mathrm{~s}$ ) for the mineral.

Figure 3(a) and 3(b) summarize the relaxation curves of four human male femurs for the collagen and the mineral, respectively. For better visibility, the samples, which cover an age between 42 and 92 years, are grouped in 10-year intervals, and the different groups are shown in different colours. As in Figure 2, the third collagen repeat distance is shown in Figure 3(a), and the lattice spacing of the (112)-reflection in Figure 3(b). Specimens were strained at rates of about $\mathrm{d} \varepsilon / \mathrm{d} t=10^{-6}$ to $10^{-5} \mathrm{~s}^{-1}$ up to fracture at $t=0$. Afterward, at $t>0$, the strain in the collagen fibrils relaxes exponentially. The average of all relaxation rates is $1 / r$ $=75 \pm 10 \mathrm{~s}$. The strain recovered is of the order of $<0 \cdot 01$ (less than $1 \%$ strain), significantly less than the total fracture strain of 0.05 . The variations between samples seem to be genuinely due to intersubject biological diversity. We could not establish

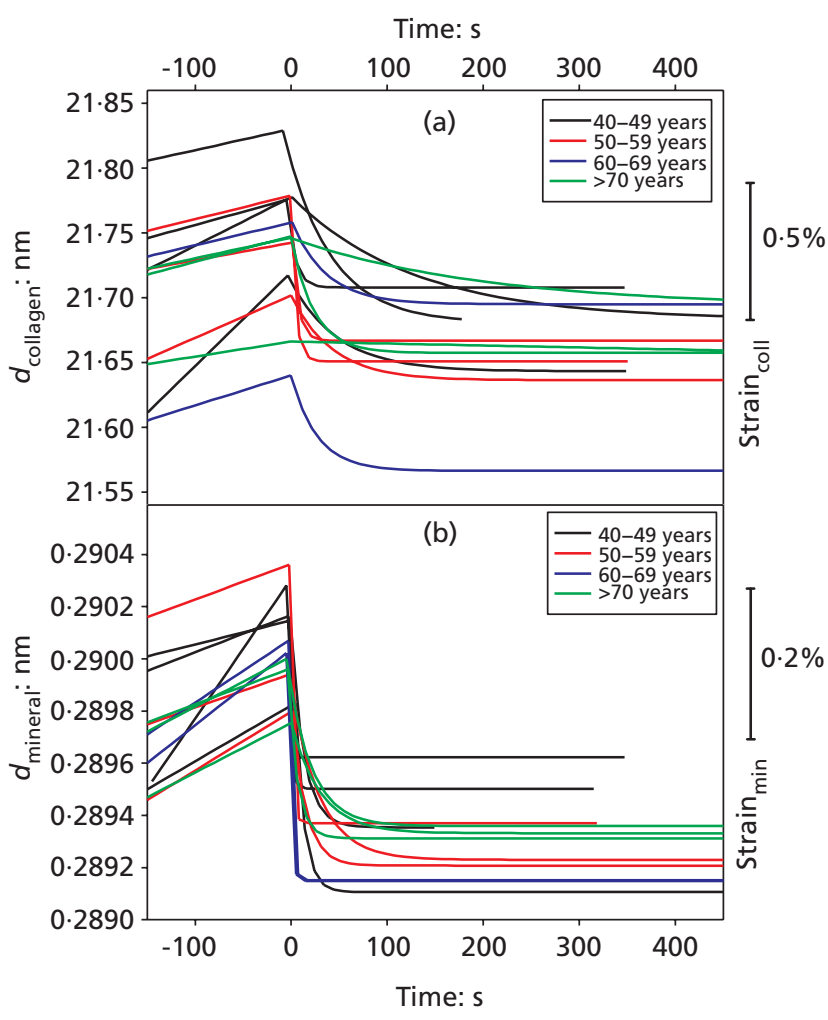

Figure 3. Relaxation curves for (a) collagen as in Figure 2(b) and (b) mineral as in Figure 2(c) for groups of different ages (shown by different colors). The $d$-period of collagen (evaluated from the third reflection) and the $d$-spacing of the mineral (evaluated from the (112)-reflection) is shown in dependence on time

any correlation with the age nor with the various biomechanical parameters investigated on bone samples, ${ }^{24,25}$ a linear regression of the relaxation times resulted in a negligible and insignificant increase with time ( $1 \mathrm{~s} /$ year with $R^{2}=0 \cdot 02$ ).

\subsection{POILS}

POILs were heated up to an order-disorder transition temperature (ODTT $=$ loss of long-range order) followed by subsequent cooling. Structural recovery can be judged either by the intensity, the half-width or the position of the peaks. We show data for the back relaxation of the position of the first (most intense) (001)peak. It follows a time-dependent recovery with

4. $d[t]=d[0]+\mathrm{c}[1-\exp \{-r t\}]$

with $d(0)$ being the lattice distance after relaxation at $t=0 \mathrm{~s}, \mathrm{c}$ a constant fit parameter describing the amount of back relaxation and $1 / r$ giving the appropriate relaxation time. 
Bioinspired, Biomimetic and Nanobiomaterials Volume 3 Issue BBN3
Timescales of self-healing in human bone tissue and polymeric ionic liquids Akbarzadeh et al.
Figure 4 shows the SAXS intensities for PIB-IL $3 b$, in dependence on the scattering vector $q=[4 \pi / \lambda] \sin \theta$, with $2 \theta$ being the scattering angle and $\lambda=0 \cdot 1542 \mathrm{~nm}$, the X-ray wavelength. During heating, the (001)-peak shifts toward smaller $q$-values (larger $d$-spacing of the lattice). Above a temperature of $70^{\circ} \mathrm{C}$, the long-range order is destroyed, visible by the disappearance of the higher-order peaks at higher $q$-values. After cooling to RT, structural reorganization starts and after $132 \mathrm{~h}$, the longrange order is completely recovered. By an anion exchange from bromide $\left(\mathrm{Br}^{-}\right)$to bis(triflouromethylsulfonyl)imide $\left(\mathrm{Tf}_{2} \mathrm{~N}\right)$, one obtains the samples PIB-IL 6a-6c. The temperature-dependent SAXS intensities of sample PIB-IL 6b (shown as Figure 5) remain similar to the ones of PIB-IL $3 b$ (loss of long-range order in this case above of $60^{\circ} \mathrm{C}$ ), but during cooling, the long-range order is immediately recovered at temperatures below $60^{\circ} \mathrm{C}$. Figure 6 shows the time-dependent shift of the normalized lattice constant for PIB-IL 3b. This back relaxation occurs with a time constant $1 / r=57 \mathrm{~h}$.

Figure 7 is a summary of the structural recovery of different POILs. In the sample series PIB-IL $3 a-3 c$, the cation is varied, whereas in the series PIB-IL 6a-6c with the numbers 3 and 6 , the anion was exchanged. Both cation and anion have a strong effect on the recovery of the long-range order: In particular, the anion exchange from bromide to $\mathrm{Tf}_{2} \mathrm{~N}$ leads, after cooling, to an immediate recovery of the structure in less than $15 \mathrm{~min}$, which is the time required to take one X-ray image. In the case of the variation of the cation, the relaxation time decreases from more than 1 week for sample PIB-IL 3 a (where even after 1 week no

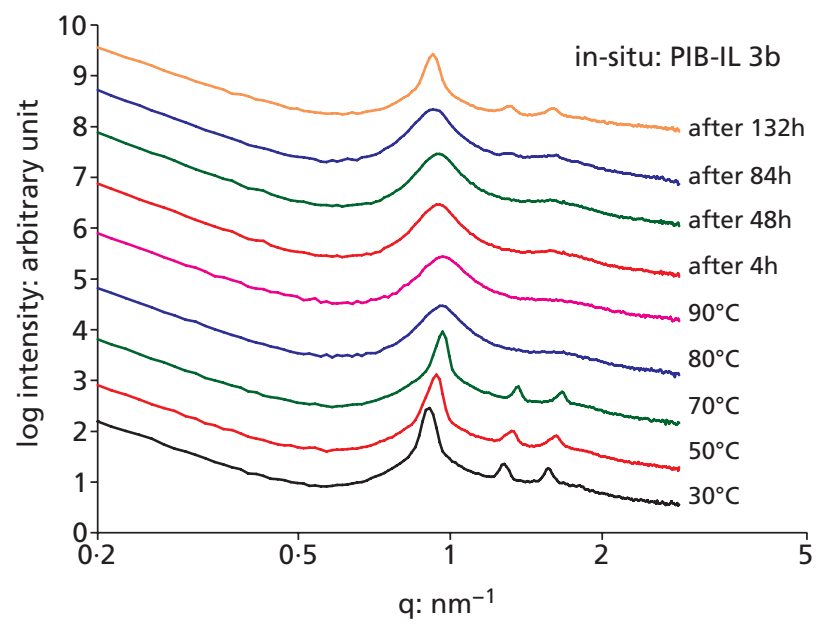

Figure 4. SAXS intensities during heating the material PIB-IL $3 \mathrm{~b}$ from 30 to $90^{\circ} \mathrm{C}$, cooling to RT and subsequent measurements for up to $132 \mathrm{~h}$. The long-range order is lost above $70^{\circ} \mathrm{C}$ and completely recovered after $132 \mathrm{~h},{ }^{22}$ visible by the disappearance and occurrence of higher-order Bragg peaks. Curves are vertically shifted for better visualization. PIB-IL, polyisobutylene-based ionic liquid; SAXS, smallangle $\mathrm{X}$-ray scattering; $\mathrm{RT}$, room temperature recovery of a long-range order was observed) to about 5 days for PIB-IL $3 b$ or less than 2 days for PIB-IL 3c, with the ionic endgroups (a) methylimidazolium, (b) pyrrolidinium and (c) triethylammonium-based, respectively.

\section{Discussion}

\subsection{Bone}

An idea of what is structurally happening can be gained from the experiments of Puxkandl et al. on tendon. ${ }^{27}$ They interpreted their diffraction data with a model in which fibrils

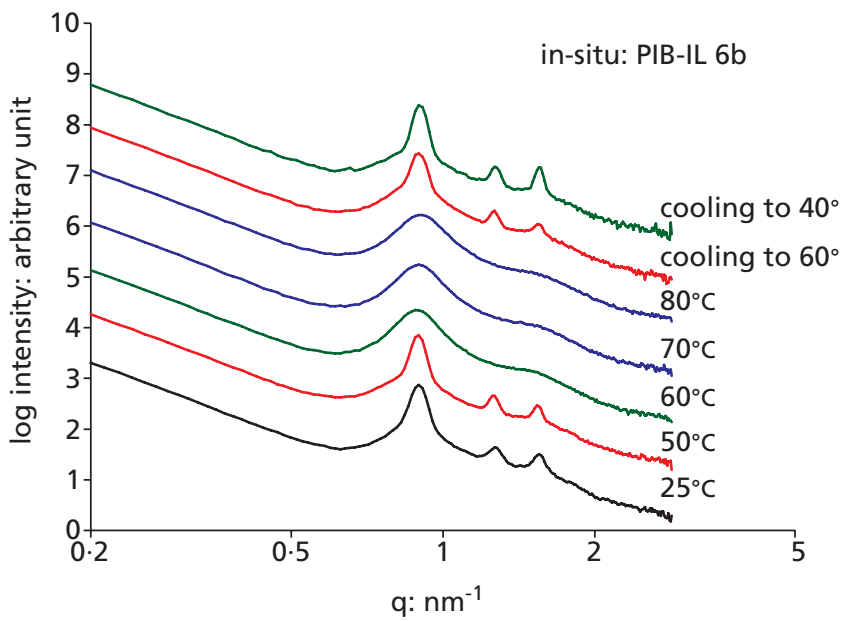

Figure 5. SAXS intensities during heating the material PIB-IL $6 \mathrm{~b}$ up to $80^{\circ} \mathrm{C}$, where long-range order is lost but recovered immediately during cooling to 60 and $40^{\circ} \mathrm{C},{ }^{23}$ visible by the disappearance and immediate occurrence of the higher-order Bragg peaks after cooling down to $60^{\circ} \mathrm{C}$. SAXS, small-angle $X$-ray scattering

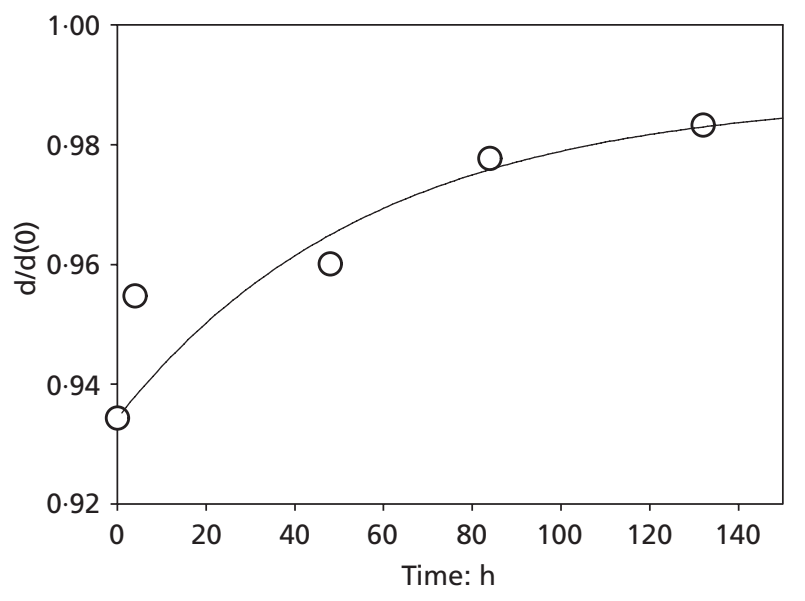

Figure 6. Strain relaxation of PIB-IL $3 b$ during cooling. The $d$-spacing is normalized by its initial value at $t=0, d(0)$, and the time-dependent recovery is shown. The time constant in Equation 4, 1/r = 57 h. PIB-IL, polyisobutylene-based ionic liquid 
Bioinspired, Biomimetic and Nanobiomaterials Volume 3 Issue BBN3
Timescales of self-healing in human bone tissue and polymeric ionic liquids

Akbarzadeh et al.

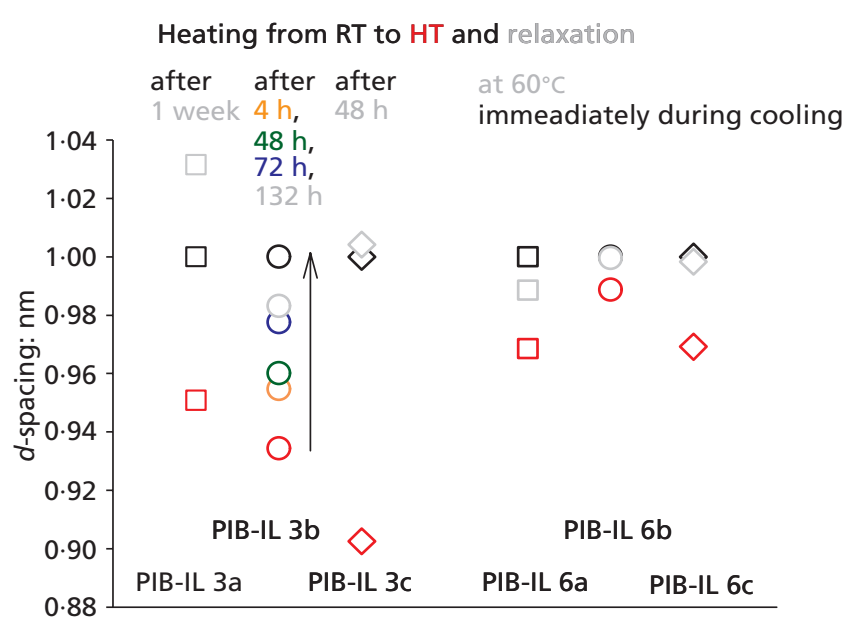

Figure 7. Different timescales of back relaxation (black symbols, original value; red symbols, $d$-spacing at high temperature; gray and colored symbols, after cooling to RT and waiting for a specific time indicated by the color): PIB-IL 3a-3c, bromide anion and ionic end groups (a) methylimidazolium (squares), (b) pyrrolidinium (circles) and (c) triethylammonium based (diamonds), PIB-IL 6a-6c, anion $\mathrm{TF}_{2} \mathrm{~N}$ and same labeling/symbols for the respective ionic end groups. $\mathrm{HT}$, high temperature; PIB-IL, polyisobutylene-based ionic liquid; RT, room temperature

and pggp-matrix are mechanically in series and arrived at $E_{\mathrm{pg}} / \eta_{\mathrm{pg}}=r_{\mathrm{pg}}=0 \cdot 4 E_{\mathrm{f}} / \eta_{\mathrm{f}}=0 \cdot 4 r_{\mathrm{f}}$, that is, in tendon, the relaxation time of the pg-matrix is 2.5 times larger than of the collagen fibrils. Since the pggp-matrix in bone consists of the same glycoproteins and proteoglycans as in tendon, but the collagen fibrils in bone are more mineralized, and therefore stiffer than in tendon, one expects that also in bone the relaxation time of the pggp-matrix to be far longer than that of the fibrils. The collagen fibrils behave almost elastically, while the pggp-matrix is viscous.

The present methodology was able to measure not only the strain in the collagen (precisely: the strain in collagen from the scattering signal of mineral crystals located in the gap zone with the $d$-period of the collagen) but also directly the strain in the hydroxyapatite (HA) inclusions. The collagen is predominantly responsible for the materials's deformation process and confers toughness to the structure, whereas the stress in the brittle HA inclusions is larger due to the higher Young's modulus. ${ }^{28}$ If bone is strained elastically, to less than the yield stress, the strains in both collagen and mineral are the same. If bone is stretched well into the plastic hardening region (1-5\% strain), the collagen relaxation time gets increasingly larger than the HA relaxation, which indicates that the pggp-matrix is responsible for the plasticity. The relaxation time in the experiment is the relaxation time of the pggp-matrix, where plasticity takes place! When the stress is relieved, the material recoils back toward its original dimensions. Not the entire viscoplastic strain in the matrix (about 5\%) can be recovered after stress relief, but only the part (about $1.5 \%$ ) driven by the elasticity of the collagen and the mineral crystals. In that sense, 'geometrically' healing is only partial: some plastic strain (per definition) persists, but the pggp-matrix and the bone are structurally as good as before.

Relaxation times of about 1 min were reported for stress relaxation of whole bone ${ }^{29,30}$ and tendon. ${ }^{31}$ The different timescales of the relaxation in $\mathrm{HA}$ inclusions and collagen suggest that the strain is redistributed and the pggp-matrix connecting collagen and HA inclusions controls the relaxation behavior. Probably, the timescale in the strain relaxation of bone of $r=0.013 \mathrm{~s}^{-1}$ has a physiological meaning: Experiments on human bone ${ }^{25,32}$ have evidenced that bone shows a ductile-to-brittle transition near physiological strain rates $\left(0 \cdot 1-0 \cdot 01 \mathrm{~s}^{-1}\right)$. The reduction in energy absorption above $0.01 \mathrm{~s}^{-1}$ is related to the amount of postyield microcracking damage. ${ }^{32}$

The self-healing under discussion is a non-biological, merely physicochemical process. Not to be confused with healing of stress fractures, and macroscopic bone traumas, taking days to weeks. Those require genuinely biological processes of remodeling and growth. Nevertheless, the fast process found provides spontaneous healing of plastically strained bone in walking or running. ${ }^{33}$

Monitoring the relaxation time $1 / r$ as a function of the proteoglycan content might allow in the future identification of the ion, which controls plasticity in the interfibrillar matrix. Such experiments on tendon, where decorin is the major proteoglycan, have shown that reduction of decorin in knock-out mice reduces the strain rate sensitivity and increases the rate of relaxation, while increase produces the opposite effect. . $^{34,35}$

\subsection{POILS}

In the PIB-IL, heating to $80^{\circ} \mathrm{C}$ produces an order-disorder phase transition, visible as a broadening of the first peak in the SAXS patterns and disappearance of higher-order peaks. The temperature increase weakens the electrostatic interaction between the ionic clusters leading to an increase in mobility of polymer chains. ${ }^{22}$ There are, however, remarkable differences between different materials: The methylimidazolium-based cation (PIB-IL 3a) is stable (up to nearly $300^{\circ} \mathrm{C}$ ), but structural recovery is slowed down or does even not take place due to thermal decomposition (see Figure 5 and Ref. ${ }^{22}$ ). A possible explanation would be a strong $\pi-\pi$ stacking between neighboring imidazolium rings, which could stabilize the structure, but can be irreversibly destroyed. In PIB-IL 3b, recovery takes some days, but only approximately 1 day for PIB-IL $3 c$, probably due to the much more flexible structure of the cation in this compound..$^{22}$ Exchanging the anionic component of the IL by the exchange of bromide with the more flexible and weakly coordinating $\mathrm{Tf}_{2} \mathrm{~N}$ anion, leads to a slight decrease of the temperature stability (to a temperature of about $60^{\circ} \mathrm{C}$ ), but much shorter times for structural recovery: No matter which cationic components of the ILs was used, the timescale for recovery was below $15 \mathrm{~min}$, the time required to collect one SAXS pattern. This is a clear 
Bioinspired, Biomimetic and Nanobiomaterials Volume 3 Issue BBN3
Timescales of self-healing in human bone tissue and polymeric ionic liquids Akbarzadeh et al. indication of the decisive role of the anion. Exchange of the anion from bromide to $\mathrm{Tf}_{2} \mathrm{~N}$ decreases the strain relaxation time, however, at the cost of a slight reduction of the stability. The influence of the cation is more by increasing the flexibility of the polymeric chain: The more flexible the chain, increasing from (a) methylimidazolium to (c) ammonium-based end groups, the higher is the probability to link to another chain and to reduce the timescale for structural recovery significantly.

Both in bone tissue and POILS, structural recovery by ionic interaction is a possible self-healing mechanism. In bone, the timescale of $1 / r=75 \mathrm{~s}$ may be biologically favorable. Bones are often loaded close to the plastic limit, for example in running. Relaxation might prevent stress fractures. In technical materials, the timescale can be varied chemically over a wide range, which allows great flexibility when using them as engineering material. The timescale is independent of the driving force, which is damage suffered. If diffusion is involved, it is of the order of $t=2 \mathrm{~L} / \mathrm{D}$, where $L$ is a characteristic length and $D$ the diffusion coefficient.

\section{Conclusions}

In elastically strained bone, the relaxation times of collagen and mineral coincide; during plastic deformation, the collagen relaxation time gets increasingly larger than the HA relaxation. This suggests that the pggp-matrix is responsible for the nanoscale plasticity of bone. The relaxation rate after fracture, $0.013 \mathrm{~s}^{-1}$, is the timescale for the self-healing of bone under natural loading conditions. Differences in the relaxation times are due to intersubject biological variability between donors, no correlation with their age was found.

Inspired by our above results on self-healing of bone, we investigated the possibility to design and optimize new, technologically interesting, self-healing materials such as POILs. To recover a long-range ordered structure in POILs, increasing the flexibility of the polymeric chains by a suited ionic end group helps, as it increases the possibility for relinking. By changing the ionic end groups and - much more effectively of - the anionic part of the IL, the timescale for structural recovery can be varied by many orders of magnitude. However, a shorter timescale for structural recovery is accompanied by a lower phase transition temperature, that is, a lower stability. Thus, varying synthesis conditions allow finding a compromise between stability and self-healing of the structure. A suitable design of the ionic interaction in POILs could be a step toward new biomimetic materials for specific applications in the future, such as self-healing of scratched coatings, or self-repair in polymeric materials. Also, one might speculate that, as chemical intervention changes the timescale in POILs, pharmaceutical intervention might change the timescale in bone.

\section{Acknowledgements}

JA and HP acknowledge the support from the Austrian Science Funds (FWF), proj. nr. I449 and the German Research Foundation
(DFG), proj. nr. PE 1732/1-2. PZ acknowledges the support provided by EPSRC (UK) under the 'Point-of-Care High Accuracy Fracture Risk Prediction' EP/K020196/1 grant. AS and WHB acknowledge the support from the German Research Foundation (DFG), proj. nr. BI 1337/8-1 within the SPP 1568 ('Design and Generic Principles of Self-Healing Materials').

\section{REFERENCES}

1. Rho, J-Y.; Kuhn-Spearing, L.; Zioupos, P. Mechanical properties and the hierarchical structure of bone. Medical Engineering \& Physics 1998, 20, 92-102.

2. Fratzl, P.; Weinkamer, R. Nature's hierarchical materials. Progress in Materials Science 2007, 52, 1263-1334.

3. Bhushan, B. Biomimetics: lessons from nature - an overview. Journal of the Royal Society Interface 2009, 367, 1445-1486.

4. Fantner, G. E.; Hassenkam, T.; Kindt, J. H.; Weaver, J. C.; Birkedal, H.; Pechenik, L.; Cutroni, J. A.; Cidade, G. A. G.; Stucky, G. D.; Morse, D. E.; Hansma, P. K. Sacrificial bonds and hidden length dissipate energy as mineralized fibrils separate during bone fracture. Nature Materials 2005, 4, 612-616.

5. Herbst, F.; Döhler, D.; Michael, P.; Binder, W. H. Self-healing polymers via supramolecular forces. Macromolecular Rapid Communications 2013, 34, 203-220.

6. Fantner, G. E.; Oroudjev, E.; Schitter, G.; Golde, L. S.; Thurner, P.; Finch, M. M.; Turner, P.; Gutsmann, T.; Morse, D. E.; Hansma, H.; Hansma, P. K. Sacrificial bonds and hidden length: unraveling molecular microstructures in tough materials. Biophysical Journal 2006, 90, 1411-1418.

7. Fratzl, P. Biomimetic materials research: what can we really learn from nature's structural materials? Journal of the Royal Society Interface 2007, 4, 637-642.

8. Peterlik, H.; Roschger, P.; Klaushofer, K.; Fratzl, P. From brittle to ductile fracture of bone. Nature Materials 2006, 5 , 51-55.

9. Gupta, H. S.; Wagermaier, W.; Zickler, G. A.; Hartmann, J.; Funari, S. S.; Roschger, P.; Wagner, D. H.; Fratzl, P. Fibrillar level fracture in bone beyond the yield point. International Journal of Fracture 2006, 139, 425-436.

10. Gupta, H. S.; Fratzl, P.; Kerschnitzki, M.; Benecke, G.; Wagermaier, W.; Kirchner, H. O. K. Evidence of an elementary process in bone plasticity with an activation enthalpy of $1 \mathrm{eV}$. Journal of the Royal Society Interface 2007, 4, 277-282.

11. Kikuchi, M.; Itoh, S.; Ichinose, S.; Shinomiya, K.; Tanaka, J. Self-organization mechanism in a bone-like hydroxyapatite/ collagen nanocomposite synthesized in vitro and its biological reaction in vivo. Biomaterials 2001, 22, 1705-1711.

12. Varley, R. S.; Shen, S.; van der Zwaag, S. The effect of cluster plasticisation on the self healing behaviour of ionomers. Polymer 2010, 51, 679-686.

13. Binder, W. H. (ed.) Self Healing Polymers: From Principles to Application. Weinheim: Wiley Verlag VCH GmbH KGaA, 2013. 
Bioinspired, Biomimetic and Nanobiomaterials Volume 3 Issue BBN3
Timescales of self-healing in human bone tissue and polymeric ionic liquids Akbarzadeh et al.
14. Wu, D. Y.; Meure, S.; Solomon, D. Self-healing polymeric materials: a review of recent developments. Progress in Polymer Science 2008, 33, 479-522.

15. Nakajima, H.; Ohno, H. Preparation of thermally stable polymer electrolytes from imidazolium-type ionic liquid derivatives. Polymer 2005, 46, 11499-11504.

16. Mecerreyes, D. Polymeric ionic liquids: broadening the properties and applications of polyectrolytes. Progress in Polymer Science 2011, 36, 1629-1648.

17. Yuan, J.; Mecerreyes, D.; Antonietti, M. Pol(ionic liquid) s: an update. Progress in Polymer Science 2013, 38, 1009-1036.

18. Domjan, A.; Erdödi, G.; Wilhelm, M.; Neidhofer, M.; Landfester, K.; Ivan, B.; Spiess, H. W. Structural studies of nanophase-separated poly(2-hydroxyethyl methacrylate)-1polyisobutylene amphiphilic conetworks by solid-state NMR and small-angle x-ray scattering. Macromolecules 2003, 36, 9107-9114.

19. Freire, M. G.; Neves, C. M. S. S.; Shimizu, K.; Bernardes, C. E. S.; Marrucho, I. M.; Coutinho, J. A. P.; Lopes, J. N. C.; Rebelo, L. P. N. Mutual solubility of water and structural/positional isomers of $\mathrm{N}$-alkylpyridinium-based ionic liquids. Journal of Physical Chemistry A 2010, 114, 15925-15934.

20. Stojanovic, A.; Kogelnik, D.; Fischer, L.; Hann, S.; Galanski, M.; Groessl, M.; Krachler, R.; Keppler, B. K. Phosphonium and ammonium ionic liquids with aromatic anions: synthesis, properties, and platinum extraction. Australian Journal of Chemistry 2010, 63, 511-524.

21. Fraser, K. J.; MacFarlane, D. R. Phosphonium-based ionic liquids: an overview. Australian Journal of Chemistry 2009, 62, 309-321.

22. Zare, P.; Stojanovic, A.; Herbst, F.; Akbarzadeh, J.; Peterlik, H.; Binder, W. H. Hierarchically nanostructured polyisobutylene-based ionic liquids. Macromolecules 2012, 45, 2074-2084.

23. Stojanovic, A.; Appiah, C.; Döhler, D.; Akbarzadeh, J.; Zare, P.; Peterlik, H.; Binder, W. H. Designing melt flow of poly(isobutylene)-based ionic liquids. Journal of Materials Chemistry A 2013, 1, 12159-12169.

24. Zioupos, P. Ageing human bone: factors affecting its biomechanical properties and the role of collagen. Journal of Biomaterials Applications 2001, 15, 187-229.

25. Zioupos, P. Accumulation of in-vivo fatigue microdamage and its relation to biomechanical properties in ageing human cortical bone. Journal of Microscopy 2001, 201, 270-278.

26. Nabarro, F. R. N.; Villiers, H. L. The Physics of Creep. London: Taylor and Francis, 1995, 67.

27. Puxkandl, R.; Zizak, I.; Paris, O.; Keckes, J.; Tesch, W.; Bernstorff, S.; Purslow, P.; Fratzl, P. Viscoelastic properties of collagen: synchrotron radiation investigations and structural model. Philosophical Transactions of the Royal Society of London 2002, B357, 191-197.
28. Nair, A. K.; Gautiere, A.; Chang, S. W.; Buehler, M. J. Molecular mechanics of mineralized collagen fibrils in bone. Nature Communications 2013, 4, 1724.

29. Iyo, T.; Yaszyzjum, M.; Sasaki, N.; Maki, Y.; Nakata, M. Anisotropic viscoelastic properties of cortical bone. Journal of Biomechanics 2004, 37, 1433-1437.

30. Iyo, T.; Sasaki, N.; Maki, Y.; Nakata, M. Mathematical description of stress relaxation of bovine femoral cortical bone. Biorheology 2006, 43, 117-132.

31. Screen, H. R. C. Investigating load relaxation mechanics in tendon. Journal of theMechanical Behavior of Biomedical Materials 2008, 1, 51-58.

32. Zioupos, P.; Hansen, U.; Currey, J. D. Microcracking damage and the fracture process in relation to strain rate in human cortical bone tensile failure. Journal of Biomechanics $\mathbf{2 0 0 8}$, 41, 2932-2939.

33. Burr, D. B.; Milgrom, C.; Fyhrie, D.; Forwood, M.; Nyska, M.; Finestone, A.; Hoshaw, S.; Saiag, E.; Simkin, A. In vivo measurement of human tibial strains during vigorous activity. Bone 1996, 18, 405-410.

34. Elliott, D. M.; Robinson, P. S.; Gimbel, J. A.; Sarver, J. J.; Abboud, J. A.; lozzo, R. V.; Soslowsky, L. J. Effect of altered matrix proteins on quasilinear viscoelastic properties in transgenic mouse tail tendons. Annals of Biomedical Engineering 2003, 31, 599-605.

35. Robinson, P. S.; Lin, T. W.; Reynolds, P. R.; Derwin, K. A.; lozzo, R. V.; Soslowsky, L. J. Strain-rate sensitive mechanical properties of tendon fascicles from mice with genetically engineered alterations in collagen and decorin. Journal of Biomechanical Engineering/Trans ASME 2004, 126, 252-257.

\section{WHAT DO YOU THINK?}

To discuss this paper, please email up to 500 words to the managing editor at bbn@icepublishing.com

Your contribution will be forwarded to the author(s) for a reply and, if considered appropriate by the editor-inchief, will be published as a discussion in a future issue of the journal.

ICE Science journals rely entirely on contributions sent in by professionals, academics and students coming from the field of materials science and engineering. Articles should be within 5000-7000 words long (short communications and opinion articles should be within 2000 words long), with adequate illustrations and references. To access our author guidelines and how to submit your paper, please refer to the journal website at www.icevirtuallibrary.com/bbn 